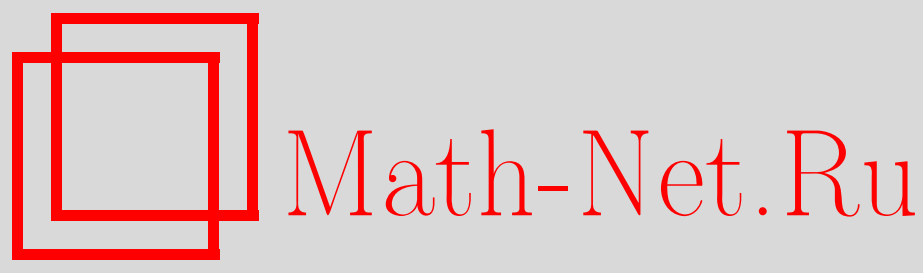

А. А. Логунов, М. А. Мествиришвили, Невозможность гравитационного коллапса, ТМФ, 2013, том 174, номep 2, 292-302

DOI: https://doi.org/10.4213/tmf8347

Использование Общероссийского математического портала Math-Net.Ru подразумевает, что вы прочитали и согласны с пользовательским соглашением http://www . mathnet.ru/rus/agreement

Параметры загрузки:

IP : 54.147 .182 .235

26 апреля 2023 г., 12:35:49

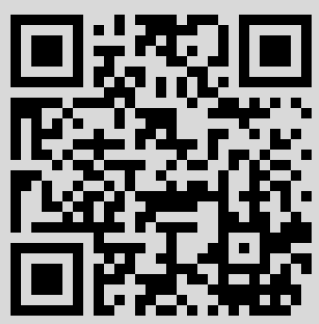




\section{НЕВОЗМОЖНОСТЬ ГРАВИТАЦИОННОГО КОЛЛАПСА}

В рамках релятивистской теории гравитации показано, что благодаря тензору гравитационного поля возникает универсальный механизм остановки процесса гравитационного сжатия тела большой массы с последующим его радиальным расширением. Это исключает гравитационный коллапс и возможность образования черных дыр.

Ключевые слова: гравитационный коллапс, принцип причинности, законы сохранения, масса гравитона.

DOI: $10.4213 / \operatorname{tmf} 8347$

Авторы статьи [1], следуя уравнениям общей теории относительности (ОТО), пришли к выводу, что массивная звезда после истощения всех термоядерных источников энергии неустойчива и переходит в стадию катастрофического сжатия происходит коллапс звезды. Эта работа позднее послужила основой для вывода о неизбежности гравитационного коллапса и существовании черных дыр [2]. Ссылаясь на статью Эйнштейна [3], которая явно отрицала возможность гравитационного коллапса, авторы монографии [2], по-видимому, не случайно завуалировали тот факт, что их результаты, изложенные в монографии, противоречат выводам Эйнштейна.

Авторы работы [1] писали: “Поскольку давления звездного вещества недостаточно для противодействия силам собственного гравитационного притяжения звезды, она, по-видимому, начнет сжиматься, так что граница $r_{\mathrm{b}}$ неизбежно будет уменьшаться до гравитационного радиуса $r_{0}$. Локальный наблюдатель, находящийся вблизи поверхности звезды, где в любом случае давление должно быть низким, увидит, что вещество уходит внутрь со скоростью, близкой к скорости света". Именно по этому поводу в статье [3] в том же 1939 г. Эйнштейн особо подчеркнул, что такого в природе не должно быть, поскольку “в противном случае частицы вещества, образующие скопление, достигнут скорости света".

Дирак в 1962 г. в статье [4] писал: “Математики могут проникать за границу шварцшильдова радиуса и попадать внутрь, но я настаиваю на том, что эта внутренняя область не является физическим пространством".

* Институт физики высоких энергий, Протвино, Московская обл., Россия. E-mail: Anatoly.Logunov@ihep.ru 
Эддингтон также не допускал возможности неограниченного сжатия тела, он по этому поводу сказал [5]: “Звезда должна излучать и излучать, сжиматься и сжиматься - до тех пор пока, как я полагаю, ее радиус не уменьшится до нескольких километров, когда притяжение становится достаточно сильным, чтобы удержать излучение, и звезда обретет, наконец, покой... Тем самым, я вынужден прийти к выводу, что это почти reductio ad absurdum (приведение к абсурду) формулы для релятивистского вырожденного газа. Возможные случайные обстоятельства могли бы предотвратить такое поведение звезды, но я хочу, чтобы был более сильный запрет на такую эволюцию звезды. Я думаю, что должен был бы существовать Закон Природы, который бы не допускал эволюции звезды столь абсурдным способом". Как мы увидим ниже, именно это и возникает в релятивистской теории гравитации (РТГ).

Заметим, что когда согласно уравнениям ОТО пробное тело в падающей системе координат достигает сферы Шварцшильда за конечное собственное время, а в другой системе координат тот же процесс происходит за бесконечное время, то совершенно очевидно, что эти системы координат связаны сингулярными преобразованиями, а такая ситуация физически недопустима.

Причина такого положения вещей в ОТО состоит в том, что известные вакуумные решения Шварцшильда, Керра и Крускала уравнения Эйнштейна удовлетворяют условиям причинности Гильберта

$$
g_{11}<0, \quad\left|\begin{array}{ll}
g_{11} & g_{12} \\
g_{21} & g_{22}
\end{array}\right|>0, \quad\left|\begin{array}{lll}
g_{11} & g_{12} & g_{13} \\
g_{21} & g_{22} & g_{23} \\
g_{31} & g_{32} & g_{33}
\end{array}\right|<0, \quad g_{00}>0
$$

не во всей области изменения переменных, что недопустимо для физического решения.

Именно поэтому Эйнштейн, следуя физической интуиции, еще в 1939 г. писал: “в реальном мире отсутствуют "шварцшильдовские сингулярности". . В противном случае частицы вещества, образующие скопление, достигнут скорости света". Но к его аргументам не прислушались, и в нарушение закона сохранения энергии и принципа причинности возникла концепция черных дыр.

В РТГ, в которой гравитационное поле рассматривается как физическое поле в пространстве Минковского, ситуация кардинально изменяется: в РТГ коллапс невозможен.

Интервал для нестатического сферически-симметричного тела имеет вид [6]

$$
d s^{2}=g_{00} d t^{2}+g_{11} d r^{2}+g_{22} d \theta^{2}+g_{33} d \phi^{2},
$$

где метрические коэффициенты $g_{00}, g_{11}$ и $g_{22}$ являются функциями радиальной переменной $r$ и времени $t$, а $g_{33}$ зависит также от угла $\theta$.

Введем следующие обозначения:

$$
\begin{aligned}
g_{00}(r, t) & =U(r, t), & g_{11}(r, t) & =-V(r, t), \\
g_{22}(r, t) & =-W^{2}(r, t), & g_{33}(r, t, \theta) & =-W^{2}(r, t) \sin ^{2} \theta .
\end{aligned}
$$

Тогда для компонент тензора $g^{\mu \nu}$ имеем

$$
\begin{aligned}
g^{00}(r, t) & =\frac{1}{U}, & g^{11}(r, t) & =-\frac{1}{V}, \\
g^{22}(r, t) & =-\frac{1}{W^{2}}, & g^{33}(r, t, \theta) & =-\frac{1}{W^{2} \sin ^{2} \theta} .
\end{aligned}
$$


Отсюда, используя выражение для интервала пространства Минковского

$$
d \sigma^{2}=d t^{2}-d r^{2}-r^{2}\left(d \theta^{2}+\sin ^{2} \theta d \phi^{2}\right),
$$

находим инвариант

$$
\gamma_{\mu \nu} g^{\mu \nu}=\frac{1}{U}+\frac{1}{V}+\frac{2 r^{2}}{W^{2}} .
$$

Кроме этого инварианта существует также инвариант

$$
\gamma^{\mu \nu} g_{\mu \nu}=U+V+\frac{2 W^{2}}{r^{2}}
$$

Гравитационное поле по величине должно быть таким, чтобы эти инварианты не были сингулярными.

Свертывая уравнение (3) с $g^{\mu \nu}$ и учитывая выражение для первого инварианта, находим общее соотношение

$$
R=2 m^{2}-\frac{m^{2}}{2}\left[\frac{1}{U}+\frac{1}{V}+\frac{2 r^{2}}{W^{2}}\right]-8 \pi T .
$$

Напомним, что величина $U$ определяет процесс замедления времени гравитационным полем.

Равенство (2) замечательно тем, что оно устанавливает прямую точную алгебраическую связь скалярной кривизны $R$, плотности $\rho$ и давления $p$ с метрическими коэфбиииентами $U, V$ и $W$.

Отсюда, в частности, следует, что при гравитационном сжатии величина $U$ не может обратиться в нуль как вне вещества, так и на поверхности тела, поскольку в противном случае скалярная кривизна $R$ стала бы бесконечной и невозможно было бы сшить внешнее решение с внутренним. Это означает, что в РТГ с необходимостью возникает остановка процесса гравитационного сжатия. Таким образом, решение уравнений гравитационного поля возможно только при ограниченном значении $R$. Далее мы увидим, что наличие тензора энергии-импульса гравитационного поля обеспечивает выполнение этого условия.

Основные уравнения РТГ имеют вид [6]

$$
\begin{aligned}
R_{\mu \nu}-\frac{m^{2}}{2}\left(g_{\mu \nu}-\gamma_{\mu \nu}\right) & =8 \pi\left(T_{\mu \nu}-\frac{1}{2} g_{\mu \nu} T\right), \\
D_{\sigma} \tilde{g}^{\lambda \sigma} & =0
\end{aligned}
$$

где $m$ - масса гравитона, $D_{\sigma}$ - ковариантная производная в пространстве Минковского. Уравнение (3) можно записать в следующей форме [6]:

$$
D_{\alpha} D_{\beta}\left(\tilde{g}^{\alpha \beta} \tilde{g}^{\epsilon \lambda}-\tilde{g}^{\epsilon \beta} \tilde{g}^{\lambda \alpha}\right)=-16 \pi g\left(T^{\epsilon \lambda}+t_{g}^{\epsilon \lambda}\right),
$$

где через $t_{g}^{\epsilon \lambda}$ обозначен тензор энергии-импульса гравитационного поля (см. приложение, формула (17)).

Перейдем теперь к анализу того, что происходит внутри вещества и вне его при гравитационном сжатии, когда термоядерные источники энергии исчерпаны [7]. Из 
уравнений РТГ (5) и (4) следует, что только в координатах Галилея имеет место закон сохранения

$$
\partial_{\lambda}\left[(-g)\left(T^{\epsilon \lambda}+t_{g}^{\epsilon \lambda}\right)\right]=0
$$

где тензор гравитационного поля $t_{g}^{\epsilon \lambda}$ определяется выражением $(5)$, записанным в координатах Галилея. В ОТО, в отличие от РТГ, закон сохранения (6) имеет место в произвольных координатах, а на месте тензора $t_{g}^{\epsilon \lambda}$ стоит не имеющий физического смысла псевдотензор. В рамках ОТО Фок [8] установил, что в гармонических координатах закон сохранения (6) приобретает физический смысл. Именно в этих координатах псевдотензор ОТО совпадает, с точностью до членов, пропорциональных массе гравитона, с тензором гравитационного поля в координатах Галилея в РТГ. Согласно (6) имеет место закон сохранения величин [9]

$$
P^{\epsilon}=\int(-g)\left(T^{\epsilon \lambda}+t_{g}^{\epsilon \lambda}\right) d S_{\lambda}
$$

При выборе гиперповерхности, которая охватывает все трехмерное пространство, выражение (7) принимает вид

$$
P^{\epsilon}=\int(-g)\left(T^{0 \epsilon}+t_{g}^{0 \epsilon}\right) d V
$$

Рассмотрим холодную нестатическую сферически-симметричную систему большой массы $\left(M>3 M_{\odot}\right)$, находящуюся в стадии, когда все термоядерные и тепловые источники энергии истощены. При гравитационном сжатии плотность энергии внутри тела возрастает при приближении к центру. Внешнее гравитационное поле такой системы согласно [6] является статическим и описывается интервалом

$$
d s^{2}=U(r) d t^{2}-V(r) d r^{2}-W^{2}(r)\left[d \theta^{2}+\sin ^{2} \theta d \phi^{2}\right] .
$$

Энергия материи и гравитационного поля согласно (8) равна

$$
P^{0}=\int_{V_{0}}(-g)\left(T^{00}+t_{g}^{00}\right) d V+\int_{V_{1}}(-g)\left(t_{g}^{00}\right) d V
$$

где $V_{0}$ - объем тела, $V_{1}$ - объем вне тела, он бесконечен. Поскольку масса гравитона $m$ очень мала, для простоты анализа мы можем положить ее равной нулю. В этом случае, оставаясь в рамках концепции полевой теории, мы должны исследовать уравнения Гильберта-Эйнштейна в гармонических координатах. Для данной задачи метрические коэффициенты в (9) для внешнего гравитационного поля согласно [8] равны

$$
U(r)=\frac{2 r-r_{g}}{2 r+r_{g}}, \quad V(r)=\frac{2 r+r_{g}}{2 r-r_{g}}, \quad W^{2}(r)=\left(r+\frac{r_{g}}{2}\right)^{2} .
$$

В нашей системе единиц $r_{g}=2 M$. Используя выражение (5) и проводя технические вычисления (см. приложение), находим

$$
16 \pi \frac{g}{\gamma} t_{g}^{00}=-\frac{r_{g}^{4}}{4 r^{6}} V^{2}(r)-\frac{3 r_{g}^{2}}{r^{5}} V(r) W(r)-\frac{r_{g}^{2}}{2 r^{6}} W^{2}(r),
$$


а для инварианта гравитационного поля $t_{g}=t_{g}^{\alpha \beta} g_{\alpha \beta}$ получим (см. приложение)

$$
32 \pi t_{g}=-\frac{4 r_{g}^{2}\left(24 r^{2}-16 r r_{g}+5 r_{g}^{2}\right)}{r^{2}\left(2 r-r_{g}\right)\left(2 r+r_{g}\right)^{3}}
$$

Из выражения (13) для инварианта гравитационного поля следует, что особенность Шварцшильда нельзя устранить выбором системы координат. Для сшивания внешнего решения с внутренним необходима ограниченность инварианта (13), что приводит к условию

$$
r>\frac{r_{g}}{2}
$$

которое доказывает, что наличие массы тела несовместимо с его точечностью [7]. Это находится в согласии с нашим выводом, полученным ранее в рамках ОТО [10]. Интегрируя выражение $(g / \gamma) t_{g}^{00}$ по объему вне тела, получим

$$
\int_{V_{1}} \frac{g}{\gamma} t_{g}^{00} d V=-\left(\frac{r_{g}}{2}\right)^{2}\left(\frac{8}{2 r_{0}(t)-r_{g}}-\frac{1}{2 r_{0}(t)}+\frac{r_{g}}{2 r_{0}^{2}(t)}+\frac{r_{g}^{2}}{8 r_{0}^{3}(t)}\right)
$$

где $r_{0}(t)$ - радиус тела.

Интеграл (8) может быть сведен к поверхностному интегралу. Его вычисление согласно [9] приводит к интегралу движения $M$

$$
P^{0}=M
$$

Учитывая (10), (14) и (15), мы приходим к следующей структуре интеграла движения:

$$
M=\int_{V_{0}} \frac{g}{\gamma}\left(T^{00}+t_{g}^{00}\right) d V-\left(\frac{r_{g}}{2}\right)^{2}\left(\frac{8}{2 r_{0}(t)-r_{g}}-\frac{1}{2 r_{0}(t)}+\frac{r_{g}}{2 r_{0}^{2}(t)}+\frac{r_{g}^{2}}{8 r_{0}^{3}(t)}\right) .
$$

В этой структуре первый член в правой части определяет энергию вещества и гравитационного поля внутри тела, тогда как второй член определяет потенциальную энергию внешнего гравитационного поля. Из выражения (14) очевидно, что если радиус тела $r_{0}(t)$ при гравитационном сжатии стремится к величине $r_{g} / 2$, то внешняя потенциальная энергия гравитационного поля стремится к отрицательной бесконечности, тогда как энергия вещества и гравитационного поля внутри тела согласно (16) неограниченно увеличивается, стремясь при гравитационном сжатии к положительной бесконечности. Но тело конечной массы в принципе не может иметь бесконечную внешнюю потенциальную энергию, поэтому такая ситуация невозможна, она противоречит принципу причинности Гильберта и закону сохранения энергии. Так проявляется свойство упругости гравитационного поля. Отсюда возникают две возможности. В первом случае благодаря упругости поля происходит остановка сжатия при некотором фиксированном радиусе, превышающем радиус Шварцшильда в гармонических координатах. Поскольку обычно считается, что статические тела большой массы не могут существовать, то эта возможность для таких тел исключается. Во втором случае после остановки гравитационного сжатия благодаря упругости поля начинается стадия радиального расширения; при этом энергия поля внутри тела уменьшается, а вместе с ней согласно (16) убывает по абсолютной величине и 
потенциальная гравитационная энергия внешнего поля. Так, именно благодаря тензору гравитационного поля $t_{g}^{\mu \nu}$ в РТГ возникает универсалъный механизм остановки процесса гравитационного сжатия тела большой массы $\left(M>3 M_{\odot}\right)$ с последующим процессом радиального расширения тела. Когда термоядерные ресурсы звезды исчерпаны, согласно РТГ из-за наличия свойства упругости гравитационного поля коллапсирующая звезда не может уйти под свой гравитационный радиус.

Аналогичный вывод в координатах кривизны имеет место и в ОТО при учете условий причинности Гильберта [10]. Этот вывод получен путем сшивания внешнего решения Шварцшильда с нестатическим решением внутри тела. Построенное таким образом физическое решение единственно, оно не имеет особенности, поскольку поверхность $r=r_{g}$ находится не в вакууме, а в веществе [11]. Это означает, что динамический процесс гравитационного сжатия тела в принципе не может описываться в ОТО только вакуумным решением уравнения Гильберта-Эйнштейна. Именно поэтому вакуумное решение Крускала не имеет никакого отношения к физическому динамическому процессу гравитационного сжатия реального тела. Решение Крускала не является физическим, поскольку оно не удовлетворяет условиям причинности Гильберта.

Объекты больших масс могут существовать, они не статические и находятся или в состоянии гравитационного сжатия, или в состоянии расширения. Поэтому гравитационный коллапс невозможен, а следовательно, невозможно и образование черных дыр.

Как видно из статьи, невозможность гравитационного коллапса возникает не только из-за наличия массы покоя гравитона, как мы предполагали ранее [6], [12], но и благодаря тензору энергии-импульса гравитационного поля. $\mathrm{K}$ аналогичному выводу, используя решение Оппенгеймера-Снайдера для гравитационного коллапса в гармонических координатах, пришел Т. Маршал [13], а также А. Митра [14].

ПРИЛОЖКННЕ

В уравнении (5)

$$
D_{\alpha} D_{\beta}\left(\tilde{g}^{\epsilon \lambda} \tilde{g}^{\alpha \beta}-\tilde{g}^{\epsilon \beta} \tilde{g}^{\lambda \alpha}\right)=-16 \pi g\left(T^{\varepsilon \lambda}+t_{g}^{\varepsilon \lambda}\right),
$$

где

$$
\begin{aligned}
-16 \pi g t_{g}^{\epsilon \lambda}= & \frac{1}{2}\left(\tilde{g}_{\nu \sigma} \tilde{g}_{\tau \mu}-\frac{1}{2} \tilde{g}_{\sigma \tau} \tilde{g}_{\mu \nu}\right)\left(\tilde{g}^{\epsilon \alpha} \tilde{g}^{\lambda \beta}-\frac{1}{2} \tilde{g}^{\epsilon \lambda} \tilde{g}^{\alpha \beta}\right) D_{\alpha} \tilde{g}^{\mu \nu} D_{\beta} \tilde{g}^{\sigma \tau}- \\
& -\tilde{g}^{\epsilon \alpha} \tilde{g}_{\sigma \tau} D_{\alpha} \tilde{g}^{\sigma \beta} D_{\beta} \tilde{g}^{\lambda \tau}-\tilde{g}^{\lambda \alpha} \tilde{g}_{\sigma \tau} D_{\alpha} \tilde{g}^{\beta \sigma} D_{\beta} \tilde{g}^{\epsilon \tau}+\tilde{g}_{\sigma \tau} \tilde{g}^{\alpha \beta} D_{\alpha} \tilde{g}^{\epsilon \sigma} D_{\beta} \tilde{g}^{\lambda \tau}+ \\
& +\frac{1}{2} \tilde{g}^{\epsilon \lambda} \tilde{g}_{\sigma \tau} D_{\alpha} \tilde{g}^{\beta \sigma} D_{\beta} \tilde{g}^{\alpha \tau}-M^{\epsilon \lambda}
\end{aligned}
$$

a

$$
M^{\epsilon \lambda}=m^{2}\left(\sqrt{-g} \tilde{g}^{\epsilon \lambda}+\tilde{g}^{\epsilon \alpha} \tilde{g}^{\lambda \beta} \gamma_{\alpha \beta}-\frac{1}{2} \tilde{g}^{\epsilon \lambda} \tilde{g}^{\alpha \beta} \gamma_{\alpha \beta}\right) .
$$

Для дальнейшего удобно ввести тензорные величины

$$
\eta^{\mu \nu}=\frac{1}{\sqrt{-\gamma}} \tilde{g}^{\mu \nu}, \quad \eta_{\mu \nu}=\sqrt{-\gamma} \tilde{g}_{\mu \nu}=\sqrt{\frac{\gamma}{g}} g_{\mu \nu}, \quad \gamma=\operatorname{det} \gamma_{\alpha \beta} .
$$


Тогда (5) и (17) примут вид

$$
D_{\alpha} D_{\beta}\left(\eta^{\alpha \beta} \eta^{\epsilon \lambda}-\eta^{\epsilon \beta} \eta^{\lambda \alpha}\right)=16 \pi \frac{g}{\gamma}\left(T^{\epsilon \lambda}+t_{g}^{\epsilon \lambda}\right),
$$

где

$$
\begin{aligned}
16 \pi \frac{g}{\gamma} t_{g}^{\epsilon \lambda}= & \frac{1}{2}\left(\eta_{\nu \sigma} \eta_{\tau \mu}-\frac{1}{2} \eta_{\sigma \tau} \eta_{\mu \nu}\right)\left(\eta^{\epsilon \alpha} \eta^{\lambda \beta}-\frac{1}{2} \eta^{\epsilon \lambda} \eta^{\alpha \beta}\right) D_{\alpha} \eta^{\mu \nu} D_{\beta} \eta^{\sigma \tau}- \\
& -\eta^{\alpha \epsilon} \eta_{\sigma \tau} D_{\alpha} \eta^{\sigma \beta} D_{\beta} \eta^{\lambda \tau}-\eta^{\alpha \lambda} \eta_{\sigma \tau} D_{\alpha} \eta^{\beta \sigma} D_{\beta} \eta^{\epsilon \tau}+\eta_{\sigma \tau} \eta^{\alpha \beta} D_{\alpha} \eta^{\epsilon \sigma} D_{\beta} \eta^{\lambda \tau}+ \\
& +\frac{1}{2} \eta^{\epsilon \lambda} \eta_{\sigma \tau} D_{\alpha} \eta^{\beta \sigma} D_{\beta} \eta^{\alpha \tau}-\bar{M}^{\epsilon \lambda}
\end{aligned}
$$

a

$$
\bar{M}^{\epsilon \lambda}=m^{2}\left(\sqrt{\frac{g}{\gamma}} \eta^{\epsilon \lambda}+\eta^{\epsilon \alpha} \eta^{\lambda \beta} \gamma_{\alpha \beta}-\frac{1}{2} \eta^{\epsilon \lambda} \eta^{\alpha \beta} \gamma_{\alpha \beta}\right) .
$$

Так как

$$
D_{\epsilon} D_{\alpha} D_{\beta}\left(\eta^{\alpha \beta} \eta^{\epsilon \lambda}-\eta^{\epsilon \beta} \eta^{\lambda \alpha}\right) \equiv 0,
$$

из (19) следует закон сохранения

$$
D_{\epsilon}\left[\frac{g}{\gamma}\left(T^{\epsilon \lambda}+t_{g}^{\epsilon \lambda}\right)\right]=0 .
$$

В координатах Галилея он имеет вид

$$
\partial_{\epsilon}\left[(-g)\left(T^{\epsilon \lambda}+t_{g}^{\epsilon \lambda}\right)\right]=0 .
$$

Для статического сферически-симметричного источника имеем

$$
\begin{aligned}
& d s^{2}=U d t^{2}-V d r^{2}-W^{2}\left(d \theta^{2}+\sin ^{2} \theta d \phi^{2}\right), \\
& d \sigma^{2}=d t^{2}-d r^{2}-r^{2}\left(d \theta^{2}+\sin ^{2} \theta d \phi^{2}\right) .
\end{aligned}
$$

Из этих выражений следует

$$
\frac{g}{\gamma}=U V \frac{W^{4}}{r^{4}}
$$

Согласно (18) и (23) имеем

$$
\begin{array}{ll}
\eta^{00}=\sqrt{\frac{V}{U}} \frac{W^{2}}{r^{2}}, \quad \eta^{11}=-\sqrt{\frac{U}{V}} \frac{W^{2}}{r^{2}}, \quad \eta^{22}=-\frac{\sqrt{U V}}{r^{2}}, \quad \eta^{33}=-\frac{\sqrt{U V}}{r^{2} \sin ^{2} \theta}, \\
\eta_{00}=\sqrt{\frac{U}{V}} \frac{r^{2}}{W^{2}}, \quad \eta_{11}=-\sqrt{\frac{V}{U}} \frac{r^{2}}{W^{2}}, \quad \eta_{22}=-\frac{r^{2}}{\sqrt{U V}}, \quad \eta_{33}=-\frac{r^{2} \sin ^{2} \theta}{\sqrt{U V}}
\end{array}
$$

а из (4) следует

$$
D_{\alpha} \eta^{\alpha \beta} \equiv \partial_{\alpha} \eta^{\alpha \beta}+\gamma_{\alpha \lambda}^{\alpha} \eta^{\lambda \beta}+\gamma_{\alpha \lambda}^{\beta} \eta^{\alpha \lambda}=0 .
$$

Отличные от нуля символы Кристоффеля для метрики $\gamma_{\mu \nu}$ равны

$$
\begin{gathered}
\gamma_{22}^{1}=-r, \quad \gamma_{33}^{1}=-r \sin ^{2} \theta, \quad \gamma_{12}^{2}=\gamma_{13}^{3}=\frac{1}{r}, \\
\gamma_{33}^{2}=-\sin \theta \cos \theta, \quad \gamma_{23}^{3}=\operatorname{ctg} \theta, \\
\gamma_{\alpha 1}^{\alpha}=\frac{2}{r}, \quad \gamma_{\alpha 2}^{\alpha}=\operatorname{ctg} \theta .
\end{gathered}
$$


Учитывая (25) и (27), из уравнения (26) для $\beta=1$ получим

$$
\frac{d}{d r}\left(\sqrt{\frac{U}{V}} \frac{W^{2}}{r^{2}}\right)=\frac{2}{r}\left(\sqrt{U V}-\sqrt{\frac{U}{V}} \frac{W^{2}}{r^{2}}\right) .
$$

Это уравнение можно записать в форме

$$
\frac{d}{d r}\left(\sqrt{\frac{U}{V}} W^{2}\right)=2 r \sqrt{U V}
$$

Легко показать, что

$$
D_{\alpha} \eta^{\alpha 2}=0, \quad D_{\alpha} \eta^{\alpha 3}=0 .
$$

Найдем теперь отличные от нуля компоненты тензора

$$
D_{\mu} \eta^{\alpha \beta}=\partial_{\mu} \eta^{\alpha \beta}+\gamma_{\mu \lambda}^{\alpha} \eta^{\lambda \beta}+\gamma_{\mu \lambda}^{\beta} \eta^{\lambda \alpha}
$$

Используя выражения для символов Кристоффеля, получим

$$
\begin{gathered}
D_{1} \eta^{00}=\frac{d}{d r}\left(\sqrt{\frac{V}{U}} \frac{W^{2}}{r^{2}}\right), \quad D_{1} \eta^{11}=-\frac{d}{d r}\left(\sqrt{\frac{U}{V}} \frac{W^{2}}{r^{2}}\right) \\
D_{1} \eta^{22}=-\frac{1}{r^{2}} \frac{d}{d r}(\sqrt{U V}), \quad D_{1} \eta^{33}=-\frac{1}{r^{2} \sin ^{2} \theta} \frac{d}{d r}(\sqrt{U V}) \\
D_{2} \eta^{12}=D_{3} \eta^{13}=\frac{1}{r}\left(\sqrt{U V}-\sqrt{\frac{U}{V}} \frac{W^{2}}{r^{2}}\right) .
\end{gathered}
$$

При $\epsilon=\lambda=0$ выражение (20) имеет вид

$$
\begin{gathered}
16 \pi \frac{g}{\gamma} t_{g}^{00}+\bar{M}^{00}=\frac{1}{2}\left(\eta_{\nu \sigma} \eta_{\tau \mu}-\frac{1}{2} \eta_{\sigma \tau} \eta_{\mu \nu}\right)\left(\eta^{0 \alpha} \eta^{0 \beta}-\frac{1}{2} \eta^{00} \eta^{\alpha \beta}\right) D_{\alpha} \eta^{\mu \nu} D_{\beta} \eta^{\sigma \tau}- \\
-2 \eta^{0 \alpha} \eta_{\sigma \tau} D_{\alpha} \eta^{\sigma \beta} D_{\beta} \eta^{0 \tau}+\eta_{\sigma \tau} \eta^{\alpha \beta} D_{\alpha} \eta^{0 \sigma} D_{\beta} \eta^{0 \tau}+\frac{1}{2} \eta^{00} \eta_{\sigma \tau} D_{\alpha} \eta^{\beta \sigma} D_{\beta} \eta^{\alpha \tau}
\end{gathered}
$$

Вычисление первого члена в правой части дает

$$
\begin{aligned}
\frac{1}{4} \frac{U}{V} & {\left[\frac{d}{d r}\left(\sqrt{\frac{V}{U}} \frac{W^{2}}{r^{2}}\right)\right]^{2}+\frac{1}{4} \frac{V}{U}\left[\frac{d}{d r}\left(\sqrt{\frac{U}{V}} \frac{W^{2}}{r^{2}}\right)\right]^{2}+\frac{V}{U} \frac{1}{r^{2}}\left(\sqrt{U V}-\sqrt{\frac{U}{V}} \frac{W^{2}}{r^{2}}\right)^{2}-} \\
& -2 \frac{W^{4}}{r^{4}}\left(\frac{d}{d r} \ln \frac{W}{r}\right)^{2}-2 \frac{W^{4}}{r^{4}} \frac{d}{d r} \ln \frac{W}{r} \frac{d}{d r} \ln \sqrt{U V} .
\end{aligned}
$$

С учетом (28) это выражение принимает вид

$$
\begin{aligned}
\frac{1}{4} \frac{U}{V} & {\left[\frac{d}{d r}\left(\sqrt{\frac{V}{U}} \frac{W^{2}}{r^{2}}\right)\right]^{2}+\frac{2}{r^{2}} \frac{V}{U}\left(\sqrt{U V}-\sqrt{\frac{U}{V}} \frac{W^{2}}{r^{2}}\right)^{2}+} \\
+ & \frac{1}{2} \frac{W^{4}}{r^{4}}\left(\frac{d}{d r} \ln \sqrt{U V}\right)^{2}-\frac{1}{2} \frac{W^{4}}{r^{4}}\left[2 \frac{d}{d r} \ln \frac{W}{r}+\frac{d}{d r} \ln \sqrt{U V}\right]^{2} .
\end{aligned}
$$

Второй член в правой части (33) равен нулю. Третий член в правой части (33) равен

$$
-\frac{U}{V}\left[\frac{d}{d r}\left(\sqrt{\frac{V}{U}} \frac{W^{2}}{r^{2}}\right)\right]^{2}
$$


Наконец, последний член в правой части (33) равен

$$
\begin{gathered}
-\frac{V}{2 U}\left[\frac{d}{d r}\left(\sqrt{\frac{U}{V}} \frac{W^{2}}{r^{2}}\right)\right]^{2}-\frac{1}{r^{2}} \frac{V}{U}\left(\sqrt{U V}-\sqrt{\frac{U}{V}} \frac{W^{2}}{r^{2}}\right)^{2}+ \\
+\frac{2 W^{2}}{r^{3}} \sqrt{\frac{V}{U}}\left(\frac{d}{d r} \ln \sqrt{U V}\right)\left(\sqrt{U V}-\sqrt{\frac{U}{V}} \frac{W^{2}}{r^{2}}\right) .
\end{gathered}
$$

Принимая во внимание (35)-(37) и учитывая (28), после некоторых преобразований получим

$$
\begin{aligned}
& 16 \pi \frac{g}{\gamma} t_{g}^{00}+\bar{M}^{00}=-\frac{3}{4} \frac{U}{V}\left[\frac{d}{d r}\left(\sqrt{\frac{V}{U}} \frac{W^{2}}{r^{2}}\right)\right]^{2}-\frac{V}{U} \frac{1}{r^{2}}\left(\sqrt{U V}-\sqrt{\frac{U}{V}} \frac{W^{2}}{r^{2}}\right)^{2}+ \\
& +\frac{1}{2} \frac{W^{4}}{r^{4}}\left[\frac{d}{d r} \ln \sqrt{U V}\right]^{2}-\frac{1}{2} \frac{W^{4}}{r^{4}}\left[2 \frac{d}{d r} \ln \frac{W}{r}+\frac{d}{d r} \ln \sqrt{U V}\right]^{2}+ \\
& +2 \frac{W^{2}}{r^{2}} \sqrt{\frac{V}{U}}\left(\frac{d}{d r} \ln \sqrt{U V}\right) \frac{1}{r}\left(\sqrt{U V}-\sqrt{\frac{U}{V}} \frac{W^{2}}{r^{2}}\right) .
\end{aligned}
$$

Можно показать, что при $\mu \neq \nu$

$$
16 \pi \frac{g}{\gamma} t_{g}^{\mu \nu}+\bar{M}^{\mu \nu}=0
$$

Для гармонического решения метрические коэффициенты вне тела имеют вид

$$
U=\frac{r-M}{r+M}, \quad V=\frac{r+M}{r-M}, \quad W=r+M .
$$

Подставляя эти выражения в (38), получим

$$
16 \pi \frac{g}{\gamma} t_{g}^{00}=-\frac{r_{g}^{4}}{4 r^{6}} V^{2}(r)-\frac{3 r_{g}^{2}}{r^{5}} V(r) W(r)-\frac{r_{g}^{2}}{2 r^{6}} W^{2}(r) .
$$

При выводе этой формулы мы положили массу гравитона равной нулю. Выражение (40) вычислено в сферических координатах. Величина $(g / \gamma) t_{g}^{00}$ не изменяется при переходе от сферических координат к декартовым, поэтому мы можем воспользоваться выражением (40) при вычислении интеграла по объему $V_{1}$ в равенстве (10). Определим теперь инвариант поля

$$
16 \pi \frac{g}{\gamma} \bar{t}_{g}+\bar{M}=\left[16 \pi \frac{g}{\gamma} t_{g}^{\mu \nu}+\bar{M}^{\mu \nu}\right] \eta_{\mu \nu}
$$

Из (20) находим

$$
16 \pi \frac{g}{\gamma} \bar{t}_{g}+\bar{M}=\frac{1}{2} \eta^{\alpha \beta}\left(\eta_{\nu \sigma} \eta_{\mu \tau}+\frac{1}{2} \eta_{\sigma \tau} \eta_{\mu \nu}\right) D_{\alpha} \eta^{\sigma \tau} D_{\beta} \eta^{\mu \nu}
$$

Учитывая равенства

$$
\begin{gathered}
\frac{1}{2} \eta^{\alpha \beta} \eta_{\nu \sigma} \eta_{\mu \tau} D_{\alpha} \eta^{\sigma \tau} D_{\beta} \eta^{\mu \nu}=-\frac{1}{2}\left(\frac{U}{V}\right)^{3 / 2} \frac{r^{2}}{W^{2}}\left[\frac{d}{d r}\left(\sqrt{\frac{V}{U}} \frac{W^{2}}{r^{2}}\right)\right]^{2}- \\
-\sqrt{\frac{U}{V}} \frac{W^{2}}{r^{2}}\left(\frac{d}{d r} \ln \sqrt{U V}\right)^{2}-\frac{4}{W^{2}} \sqrt{\frac{V}{U}}\left(\sqrt{U V}-\sqrt{\frac{U}{V}} \frac{W^{2}}{r^{2}}\right)^{2} \\
\frac{1}{4} \eta^{\alpha \beta} \eta_{\sigma \tau} \eta_{\mu \nu} D_{\alpha} \eta^{\sigma \tau} D_{\beta} \eta^{\mu \nu}=-\sqrt{\frac{U}{V}} \frac{W^{2}}{r^{2}}\left[\frac{d}{d r} \ln \sqrt{U V}+2 \frac{d}{d r} \ln \frac{W}{r}\right]^{2}
\end{gathered}
$$


из (42) получим

$$
\begin{aligned}
16 \pi \frac{g}{\gamma} \bar{t}_{g}+\bar{M}= & -\frac{1}{2}\left(\frac{U}{V}\right)^{3 / 2} \frac{r^{2}}{W^{2}}\left[\frac{d}{d r}\left(\sqrt{\frac{V}{U}} \frac{W^{2}}{r^{2}}\right)\right]^{2}- \\
& -4 \sqrt{\frac{V}{U}} \frac{1}{W^{2}}\left(\sqrt{U V}-\sqrt{\frac{U}{V}} \frac{W^{2}}{r^{2}}\right)^{2}-\sqrt{\frac{U}{V}} \frac{W^{2}}{r^{2}}\left[\frac{d}{d r} \ln \sqrt{U V}\right]^{2}- \\
& -\sqrt{\frac{U}{V}} \frac{W^{2}}{r^{2}}\left[\frac{d}{d r} \ln \sqrt{U V}+2 \frac{d}{d r} \ln \frac{W}{r}\right]^{2}
\end{aligned}
$$

где

$$
\bar{M}=m^{2} \frac{W^{2}}{r^{2}}\left[4 \sqrt{U V}-\sqrt{\frac{V}{U}}-\sqrt{\frac{U}{V}}-2 \sqrt{U V} \frac{W^{2}}{r^{2}}\right] .
$$

Приведем компоненты тензора $Q^{\epsilon \lambda}$ :

$$
Q^{\epsilon \lambda}=D_{\alpha} D_{\beta}\left(\eta^{\alpha \beta} \eta^{\epsilon \lambda}-\eta^{\epsilon \alpha} \eta^{\lambda \beta}\right) .
$$

Если интервал имеет форму (23), то

$$
\begin{aligned}
Q^{00}= & -\sqrt{\frac{U}{V}} \frac{W^{2}}{r^{2}} \frac{d^{2}}{d r^{2}}\left(\sqrt{\frac{V}{U}} \frac{W^{2}}{r^{2}}\right)-\frac{2}{r} \sqrt{U V} \frac{d}{d r}\left(\sqrt{\frac{V}{U}} \frac{W^{2}}{r^{2}}\right) \\
Q^{11}= & \left(\sqrt{\frac{U}{V}} \frac{W^{2}}{r^{2}}\right) \frac{d^{2}}{d r^{2}}\left(\sqrt{\frac{U}{V}} \frac{W^{2}}{r^{2}}\right)+4 \frac{\sqrt{U V}}{r^{2}}\left(\sqrt{U V}-\sqrt{\frac{U}{V}} \frac{W^{2}}{r^{2}}\right)+ \\
& +2 \frac{\sqrt{U V}}{r} \frac{d}{d r}\left(\sqrt{\frac{U}{V}} \frac{W^{2}}{r^{2}}\right)-\left[\frac{d}{d r}\left(\sqrt{\frac{U}{V}} \frac{W^{2}}{r^{2}}\right)\right]^{2}- \\
& -\frac{2}{r^{2}}\left(\sqrt{U V}-\sqrt{\frac{U}{V}} \frac{W^{2}}{r^{2}}\right)^{2}, \\
Q^{22}= & \frac{1}{r^{2}} \sqrt{\frac{U}{V}} \frac{W^{2}}{r^{2}} \frac{d^{2}}{d r^{2}}(\sqrt{U V})-\frac{2 \sqrt{U V}}{r^{4}}\left(\sqrt{U V}-\sqrt{\frac{U}{V}} \frac{W^{2}}{r^{2}}\right)+ \\
Q^{33}= & \frac{1}{\sin ^{2} \theta} Q^{22}, \quad Q^{\mu \nu}=0, \quad \mu \neq \nu .
\end{aligned}
$$

Подставляя в (44) метрические коэффициенты (39), находим инвариант гравитационного поля $t_{g}=t_{g}^{\mu \nu} g_{\mu \nu}$ :

$$
32 \pi t_{g}=-\frac{4 r_{g}^{2}\left(24 r^{2}-16 r r_{g}+5 r_{g}^{2}\right)}{r^{2}\left(2 r-r_{g}\right)\left(2 r+r_{g}\right)^{3}} .
$$

При нахождении этого выражения мы положили массу гравитона равной нулю.

Учитывая в (47) метрические коэффициенты (39), получим

$$
\begin{gathered}
Q^{00}=-\frac{2 M^{2}}{r^{6}} V^{2}\left(7 r^{2}-8 M r+3 M^{2}\right), \\
Q^{11}=\frac{2 M^{2}}{r^{4}}, \quad Q^{22}=-\frac{2 M^{2}}{r^{6}}, \quad Q^{33}=-\frac{2 M^{2}}{r^{6} \sin ^{2} \theta}, \\
Q^{\mu \nu} \eta_{\mu \nu}=-\frac{2 M^{2}}{r^{4}\left(r^{2}-M^{2}\right)}\left[6 r^{2}-8 M r+5 M^{2}\right] .
\end{gathered}
$$


Благодарности. В заключение авторы выражают благодарность С. С. Герштейну, В.А. Петрову, А. П. Самохину, Н. Е. Тюрину за интерес к обсуждаемой теме и ценные замечания.

\section{Список литературы}

[1] Ю. Оппенгеймер, Г. Снайдер, “О безграничном гравитационном сжатии”, Алъберт Эйнштейн и теория гравитации, Сб. статей, ред. Е. С. Куранский, Мир, М., 1979, 353-361.

[2] Дж. Уилер, Б. Гаррисон, М. Вакано, К. Торн, Теория гравитации и гравитаиионный коллапс, Мир, М., 1967.

[3] А. Эйнштейн, "О стационарных системах, состоящих из многих гравитирующих частиц и обладающих сферической геометрией”, ст. 119, Собрание научных трудов, т. II: Работы по теории относительности 1921-1955 г2., Наука, М., 1966, 514-531.

[4] P. A. M. Dirac, Proc. Roy. Soc. London Ser. A, 270:1342 (1962), 354-356.

[5] A. S. Eddington, Observatory, 58 (1935), 37-39.

[6] А.А. Логунов, Релятивистская теория гравитации, Наука, М., 2011.

[7] А. А. Логунов, М. А. Мествиришвили, ТМФ, 171:1 (2012), 150-153.

[8] В. А. Фок, Теория пространства, времени и тяготения, Физматлит, М., 1961.

[9] Л. Д. Ландау, Е. М. Лифшиц, Теоретическая физика, т. 2: Теория поля, Наука, М., 2001.

[10] А. А. Логунов, М. А. Мествиришвили, ТМФ, 170:3 (2012), 489-496.

[11] Дж. Синг, Общая теория относительности, ИЛ, М., 1963.

[12] С. С. Герштейн, А. А. Логунов, М. А. Мествиришвили, ТМФ, 161:2 (2009), 295-304.

[13] T. Marshall, Fields tell matter how to move, arXiv: 1103.6168.

[14] A. Mitra, Astron. Space Sci., 332:1 (2011), 43-48. 